\title{
EZH2 inhibition suppresses bladder cancer cell growth and metastasis via the JAK2/STAT3 signaling pathway
}

\author{
ZHIYUAN CHEN, YANG DU, XIUHENG LIU, HUI CHEN, \\ XIAODONG WENG, JIA GUO, MIN WANG, XIAO WANG and LEI WANG \\ Department of Urology, Renmin Hospital of Wuhan University, Wuhan, Hubei 430060, P.R. China
}

Received June 8, 2018; Accepted April 3, 2019

DOI: $10.3892 / \mathrm{ol} .2019 .10359$

\begin{abstract}
The aim of the current study was to investigate the role of enhancer of zeste 2 polycomb repressive complex 2 subunit $(\mathrm{EZH} 2)$ in the progression of bladder cancer. Human bladder cancer tissue samples were analyzed by immunohistochemistry, and the association between the clinicopathological parameters and EZH2 expression was analyzed. The proliferation, apoptosis and migration ability of the human bladder cancer cell lines E-J and 5637 with or without the EZH2 inhibitor UNC1999 was investigated. The effect of UNC1999 was further explored in a xenograft model of nude mice. The in vivo and in vitro expression levels of $\mathrm{EZH} 2$, janus kinase 2, signal transducer and activator of transcription 3 and their phosphorylated forms were examined by western blotting. The expression levels of EZH2, JAK2 and STAT3 were increased in bladder cancer tissue compared with normal adjacent tissue. Furthermore, the expression of EZH2 was increased in tumors with a higher TNM Classification of Malignant Tumors stage and histological grade compared with tumors with a lower stage and grade. The human bladder cancer cell lines E-J and 5637 treated with UNC1999 demonstrated reduced cell proliferation, apoptosis and migration compared with cells treated without UNC1999. Additionally, EZH2 may promote the proliferation and migration of bladder cancer via the JAK2/STAT3 pathway. EZH2 may serve an important role in the proliferation and migration of human bladder cancer cells, and may aid in the development of novel treatment strategies for bladder cancer.
\end{abstract}

\section{Introduction}

Bladder cancer is the most common tumor among genitourinary cancers and the fourth most common cancer among

Correspondence to: Dr Lei Wang, Department of Urology, Renmin Hospital of Wuhan University, 238 Jiefang Road, Wuhan, Hubei 430060, P.R. China

E-mail: drwanglei@whu.edu.cn

Key words: bladder cancer, enhancer of zeste 2 polycomb repressive complex 2 subunit, janus kinase 2, signal transducer and activator of transcription 3 males (1). Bladder cancer has a high incidence and mortality rate among the malignant tumors of the genitourinary tract (2). Furthermore, bladder cancer is more prevalent than other urinary tract carcinomas (3). Cancer statistical data have indicated an estimated 549,393 new cases and 199,922 mortalities from bladder cancer worldwide in 2018 (4). The majority of the newly diagnosed tumors are superficial and may be treated by transurethral resection (5). However, a large number of patients have a high rate of tumor recurrence following the first surgery (6). The mechanisms underlying the development of bladder cancer have not been thoroughly elucidated. Therefore, the identification of the molecular mechanisms involved in the progression of bladder tumors may aid the development of novel diagnostic and therapeutic interventions. The signal transducer and activator of transcription 3 (STAT3) signaling pathway is implicated in the progression of lung cancer (7). STAT3 is a cytoplasmic transcription factor which is expressed in response to a large number of cytokines and growth factors (8). A previous study suggested that STAT3 may promote the growth of tumor cells and inhibit tumor cell apoptosis (9). STAT3 is activated by the phosphorylation of conserved tyrosine and serine residues in its C-terminal domains by janus kinase (JAK) proteins (9). The phosphorylation of proteins in the JAK2/STAT3 pathway may result in the growth and proliferation of bladder cancer cells (10). The enhancer of zeste 2 polycomb repressive complex 2 subunit (EZH2) is a member of the polycomb group proteins (11). EZH2 serves important roles in embryonic stem cell pluripotency and self-renewal $(12,13)$. Furthermore, EZH2 expression was upregulated in different types of malignant tumors with poor prognosis, including prostate, bladder, renal and breast carcinomas (14-17). Previous studies revealed that EZH2 may be a marker of aggressive urological neoplasms $(18,19)$. However, the biological effect of EZH2 in bladder cancer and the associations between EZH2 and the JAK2/STAT3 signaling pathway have not been elucidated. The aim of the current study was to investigate the effects of EZH2 on bladder carcinogenesis and to explore its mechanism in human bladder cancer cells.

\section{Materials and methods}

Drugs and reagents. The EZH2 inhibitor UNC1999 was purchased from Selleck Chemicals (Shanghai, China) and 
was dissolved in dimethyl sulfoxide (DMSO; concentrations used in experiments, 0.1, 1, 10 and $100 \mu \mathrm{M})$. Antibodies against phospho-JAK2 (1:1,000 dilution; cat. no. 3771S), JAK2 (1:1,000 dilution; cat. no. 3230S), phospho-STAT3 (1:1,000 dilution; cat. no. 9145S), STAT3 (1:1,000 dilution; cat. no. 9139S), EZH2 (1:1,000 dilution; cat. no. 5246S) and GAPDH (1:3,000 dilution; cat. no. 5174S), as well as horseradish peroxidase-conjugated goat anti-rabbit and anti-mouse IgG (1:2,000 dilution; cat. nos. 7074S and 7076S), were purchased from Cell Signaling Technology, Inc. (Boston, MA, USA).

Clinical specimens. Human bladder tumors and corresponding adjacent normal tissues were collected from patients who underwent partial or radical cystectomy for urothelial carcinomas of the bladder at the Department of Urology of Renmin Hospital of Wuhan University (Wuhan, China) between June 2015 and March 2017. The study was approved by the Institutional Ethics Committee of Renmin Hospital of Wuhan University. Each participant provided signed informed consent prior to participation in the present study. Patients or their legal surrogate decision makers provided signed informed consent for the surgical procedures. All specimens were anonymized. Inclusion criteria were the following: i) $\leq 75$ years; ii) histologically confirmed bladder cancer; iii) no severe major organ dysfunction; and iv) no prior cancer chemotherapy. Exclusion criteria were the following: i) $\geq 76$ years; ii) severe major organ dysfunction; and iii) prior cancer chemotherapy. The clinical information of the patients, including sex, age, smoking history, the diameter and differentiation of the tumor, lymph node metastasis, stage of TNM, histological grade, chemotherapy received and EZH2 expression status, were recorded. Half of the specimens were removed and fixed in $4 \%$ paraformaldehyde at room temperature for 1 week, followed by routine paraffin embedding, and the remaining half were preserved in liquid nitrogen at $-196^{\circ} \mathrm{C}$. All of the specimens were classified as bladder carcinoma or normal tissue by histological identification. Tumor stage and grade were determined according to the Union for International Cancer Control (UICC) guidelines (20).

Cell lines and cell culture. The human bladder cancer cell lines E-J and 5637 were purchased from the The Cell Bank of Type Culture Collection of Chinese Academy of Sciences (Shanghai, China). E-J and 5637 cells were authenticated by STR profiling. E-J and 5637 cells were cultured at $37^{\circ} \mathrm{C}$ with $5 \% \mathrm{CO}_{2}$. in RPMI 1640 medium (Thermo Fisher Scientific, Inc., Waltham, MA, USA), supplemented with $10 \%$ fetal bovine serum (FBS; ScienCell Research Laboratories, Inc., San Diego, CA, USA), $0.1 \mathrm{mg} / \mathrm{ml}$ streptomycin and $100 \mathrm{U} / \mathrm{ml}$ penicillin. The medium was replaced every $48 \mathrm{~h}$.

Cell viability assay. E-J and 5637 cells were plated into 96-well plates at a density of $5 \times 10^{3}$ cells/well. Following $24 \mathrm{~h}$ of culture at $37^{\circ} \mathrm{C}$, the cells were treated with different concentrations of UNC1999 $(0.1,1,10$ and $100 \mu \mathrm{M})$ for different times $(24,72$ and $120 \mathrm{~h})$ and subsequently incubated with $10 \mu \mathrm{l}$ of MTT dye/well for $4 \mathrm{~h}$. Following the MTT incubation, the purple formazan crystals were dissolved by the addition of $150 \mu 1$ DMSO per well. Cell viability was subsequently analyzed at a wavelength of $490 \mathrm{~nm}$.
Apoptosis assay. E-J and 5637 cells were incubated with or without UNC1999 $(100 \mu \mathrm{M})$. The control group was treated with DMSO. for $72 \mathrm{~h}$. The cells were collected and washed twice with PBS and resuspended with $150 \mathrm{ml}$ binding buffer (included in the Annexin V-FITC Apoptosis Detection kit; cat. no. C1062M; Beyotime Institute of Biotechnology). The cells were subsequently incubated with $10 \mu \mathrm{l}$ Annexin V-fluorescein isothiocyanate (FITC) and $5 \mu \mathrm{l}$ propidium iodide for $5 \mathrm{~min}$ at room temperature in the dark. The Annexin V-FITC Apoptosis Detection kit was used according to the manufacturer's protocol. Apoptotic cells were subsequently analyzed using a flow cytometer (BD Biosciences, San Jose, CA, USA). Analysis was performed using the BD FACSuite software (version 1.0; BD Biosciences).

Wound healing assay. E-J and 5637 cells $\left(5 \times 10^{5}\right)$ were plated in six-well plates. Following a $24 \mathrm{~h}$ incubation period at $37^{\circ} \mathrm{C}$ with $5 \% \mathrm{CO}_{2}$. wounds were made in each well using a $200 \mu \mathrm{l}$ pipette tip. The cells were washed three times with PBS and incubated with UNC1999 $(100 \mu \mathrm{M})$ at $37^{\circ} \mathrm{C}$ for $24 \mathrm{~h}$. The wound areas were subsequently quantified using a microscope.

Cell migration assays. A total of $5 \times 10^{4} \mathrm{E}-\mathrm{J}$ and 5637 cells were plated in the upper chambers of Transwell plates (24 wells; $8 \mu \mathrm{m}$ pore size with polycarbonate membrane; Corning Inc., Corning, NY, USA) in $100 \mu 1$ serum-free RPMI 1640 medium. RPMI 1640 medium containing 10\% FBS and UNC1999 $(100 \mu \mathrm{M})$ was plated in the lower chambers. Following incubation at $37^{\circ} \mathrm{C}$ for $24 \mathrm{~h}$, the migratory cells were fixed with methanol at room temperature for $6 \mathrm{~h}$ and stained with $0.2 \%$ crystal violet (Beyotime Institute of Biotechnology). The numbers of stained cells in five randomly selected fields were counted using a light microscope (magnification, x400; Leica Microsystems, Ltd., Milton Keynes, UK).

Immunohistochemistry. The expression of EZH2 was assessed by immunohistochemical staining. The bladder cancer tissues and para-carcinoma tissues were cut into $4-\mu \mathrm{m}$ thick sections. Endogenous peroxidase activity was inhibited with $3 \%$ hydrogen peroxide at $37^{\circ} \mathrm{C}$ for $10 \mathrm{~min}$. The sections were subsequently treated with 1:50 normal horse serum (cat. no. 16050130; Thermo Fisher Scientific, Inc.) in tris-buffered saline for $30 \mathrm{~min}$ at $37^{\circ} \mathrm{C}$. Tissue sections were incubated with primary antibody directed against EZH2 overnight at $4^{\circ} \mathrm{C}$. The sections were subsequently washed three times with PBS. The sections were incubated with a horseradish peroxidase-labeled secondary antibody for $30 \mathrm{~min}$ at $20^{\circ} \mathrm{C}$. The sections were incubated with 3,3'-diaminobenzidine for $2 \mathrm{~min}$ at $20^{\circ} \mathrm{C}$. Tissues were observed under a light microscope (magnification, $\mathrm{x} 400$ ) and images were captured. Immunostained sections were evaluated by two experienced pathologists under blinded conditions.

Reverse transcription-semi-quantitative polymerase chain reaction (RT-qPCR). RT-qPCR was used to assess the EZH2 mRNA level in clinical specimens. Total RNA was extracted from the aforementioned clinical specimens and cells using TRIzol ${ }^{\circledR}$ reagent (Invitrogen; Thermo Fisher Scientific, Inc.). EZH2 levels were assessed using a Roche LightCycler 480 (Roche Diagnostics GmbH, Mannheim, Germany) and 
A

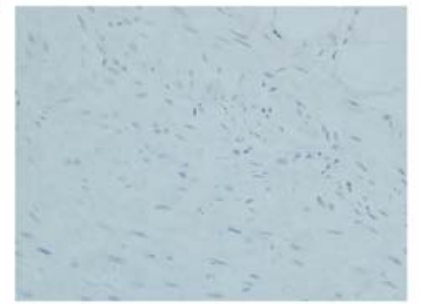

C

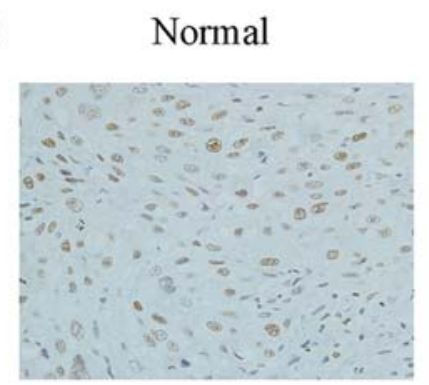

High expression
B

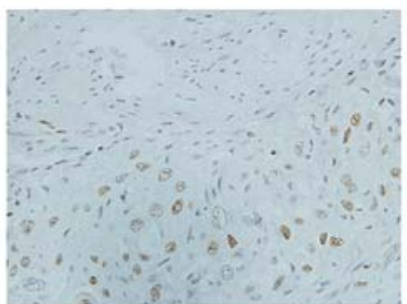

D

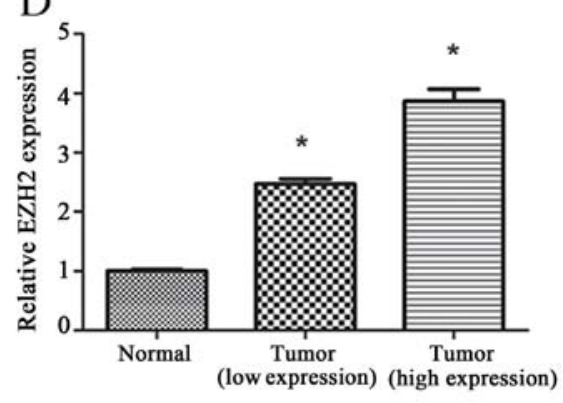

E

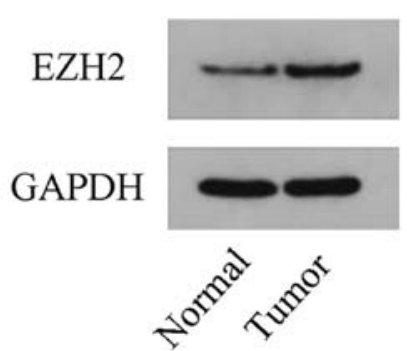

F

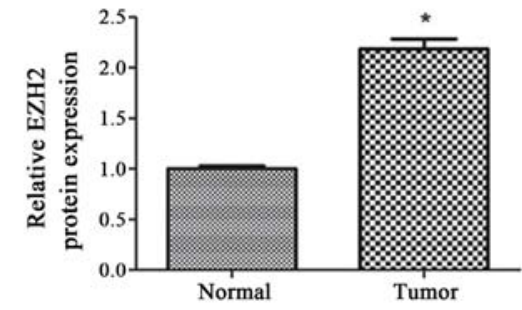

Figure 1. EZH2 levels in bladder cancer. Immunohistochemical analysis of EZH2 in (A) normal bladder tissues, (B) low expression and (C) high expression bladder tumor tissues (magnification, $\mathrm{x} 400$ ). (D) Reverse transcription-semi-quantitative polymerase chain reaction analysis of the expression level of EZH2 in human bladder cancer tissues from 34 patients. (E) Western blot analysis of the expression level of EZH2 in human bladder cancer tissues. (F) Quantification of protein bands in human bladder cancer tissues. ${ }^{*} \mathrm{P}<0.01$ vs. normal group. EZH2, enhancer of zeste 2 polycomb repressive complex 2 subunit.

BeyoFast $^{\mathrm{TM}}$ SYBR Green qPCR Mix kit (cat. no. D7260; Beyotime Institute of Biotechnology, Haimen, China) according to the manufacturer's protocols. GAPDH was used as an endogenous reference gene to analyse the relative gene expression levels. The thermocycling conditions were as follows: 1 cycle of $94^{\circ} \mathrm{C}$ for $3 \mathrm{~min}$, followed by 35 cycles of $94^{\circ} \mathrm{C}$ for $5 \mathrm{sec}, 56^{\circ} \mathrm{C}$ for $30 \mathrm{sec}$ and $72^{\circ} \mathrm{C}$ for $30 \mathrm{sec}$, and a final extension at $60^{\circ} \mathrm{C}$ for $30 \mathrm{sec}$. The quantification cycle fluorescence value $(\mathrm{Cq})$ was calculated using SDS software (version 2.1; Applied Biosystems; Thermo Fisher Scientific, Inc.). The expression levels were analysed according to the $2^{-\Delta \Delta \mathrm{Cq}}$ method (21). All experiments were performed in triplicate. The appearance of a single peak in the melting curve implicated the specificity of the PCR products. The primer sequences were as follows: EZH2 forward, 5'-GACCCTGAC CTCTGTCTTACTT-3', and reverse, 5'-GATGGTGCCAGG CAATAGATG-3'; and GAPDH forward, 5'-AGGTCGGTG TGAACGGATTTG-3', and reverse, 5'-TGTAGACCATGT AGTTGAGGTCA-3'.

Flank xenograft model. The Institutional Animal Care and Use Committee of Wuhan University approved the experimental protocols and supervised the care of animals and experimental procedures. Nude mice (Balb/c nu/nu) were purchased from the Animal Center of Wuhan University. BALB/c nude mice ( $\mathrm{n}=10$; male) were used for in vivo experiments. The mice were bred and experiments were performed in laminar flow cabinets under specific-pathogen-free conditions in the Laboratory Animal Center of Renmin Hospital of Wuhan University. Nude mice were kept at the College Laboratory Animal Center of Conventional Breeding at a temperature of $25-27^{\circ} \mathrm{C}$ with a relative humidity of $45-50 \%$, in an aseptic environment, with adequate illumination (12-h light/dark cycle), moisture and feed. The mice had free access to food and water. Preliminary in vivo experiments revealed that the tumorigenic effect of E-J cells was better than that of 5637 cells; therefore, E-J cells were used in the in the in vivo experiments. A total of $5 \times 10^{6}$ E-J cells were inoculated in the flank region of six-week-old nude mice (weight, 16-20 g). Two perpendicular diameters ( $a$, the largest; $b$, the smallest) of the tumor were measured once per week with calipers for 5 weeks of monitoring. Tumor volume (V) was calculated using the following formula: $\mathrm{V}=\mathrm{a} \times \mathrm{b}^{2}$ x 0.5 (22). UNC1999 $(50 \mathrm{mg} / \mathrm{kg})$ and saline in equal volumes were injected intraperitoneally twice per week when the mean tumor volume reached $\sim 100 \mathrm{~mm}^{3}$. Tumor growth rate was plotted and analyzed using GraphPad Prism software (version 5.0; GraphPad Software, Inc., La Jolla, CA, USA). 
Table I. Clinicopathological features of the 34 patients with bladder cancer and their associations with EZH2 expression.

\begin{tabular}{lcccc}
\hline & \multicolumn{4}{c}{$\begin{array}{c}\text { EZH2 } \\
\text { expression }\end{array}$} \\
\cline { 3 - 4 } Characteristic & No. of patients & Low & High & P-value \\
\hline Age (years) & 12 & 4 & 8 & 0.86 \\
$\quad<60$ & 22 & 8 & 14 & \\
$>60$ & & & & 0.714 \\
Sex & 24 & 8 & 16 & \\
Male & 10 & 4 & 6 & \\
Female & & & & $0.026^{\mathrm{a}}$ \\
Histological stage & 14 & 8 & 6 & \\
Ta-T1 & 20 & 4 & 16 & \\
T2-T4 & & & & $<0.001^{\mathrm{a}}$ \\
Histological grade & 15 & 10 & 5 & \\
G1 & 19 & 2 & 17 & \\
G2-G3 & & & &
\end{tabular}

P-values were calculated using a Fisher's exact test. ${ }^{\text {aStatistically }}$ significant. The mean of the relative expression of EZH2 in tissues of all paired samples was used as the cut-off value to determine high and low expression groups. EZH2, enhancer of zeste 2 polycomb repressive complex 2 subunit; Ta, non-invasive papillary carcinoma.

Western blotting. E-J and 5637 cells or tumor tissues from mice were dissociated using the Total Protein Extraction kit (Wuhan Goodbio Technology Co., Ltd., Wuhan, China) according to the manufacturer's instructions, and the extracted protein was examined by western blotting. Protein concentrations were assessed using a bicinchoninic acid assay prior to loading. A total of $40 \mu \mathrm{g}$ protein/lane from each sample were separated via SDS-PAGE on a $10 \%$ gel. The separated proteins were subsequently transferred onto nitrocellulose membranes and blocked for $2 \mathrm{~h}$ with $5 \%$ nonfat milk in Tris-buffered saline and Tween-20 (TBST) buffer at room temperature. The membranes were incubated with the primary polyclonal antibodies against EZH2, p-JAK2, JAK2, p-STAT3, STAT3 and GAPDH overnight at $4^{\circ} \mathrm{C}$. Membranes were washed three times with TBST. Following the primary incubation, membranes were incubated with horseradish peroxidase-labeled secondary antibodies $(1: 2,000)$. The membranes were subsequently washed three times with TBST. The protein bands were visualized using an enhanced chemiluminescence detection kit (EMD Millipore, Billerica, MA, USA). The band intensity was quantified using ImageJ software (version 2.1; National Institutes of Health, Bethesda, MD, USA).

Statistical analysis. Data are presented as the means \pm standard error of the mean. SPSS software (version 17; SPSS, Inc., Chicago, IL, USA) was used for statistical analysis. Differences in values and percentages among groups were compared using a paired t-test, $\chi^{2}$ test, Fisher's exact test or one-way analysis of variance followed by Dunnett's multiple comparison test. All experiments were repeated at least three times. $\mathrm{P}<0.05$ was considered to indicate a statistically significant difference.

\section{Results}

Expression levels of EZH2 in bladder cancer. In order to investigate whether the expression level of EZH2 was associated with the progression of bladder cancer, the expression levels of $\mathrm{EZH} 2$ in bladder carcinoma tissue and adjacent non-neoplastic parenchyma were analyzed by immunohistochemical staining. The mean of the relative expression of EZH2 in tissues of all paired samples was used as the cut-off value to determine high and low expression groups. The expression levels of EZH2 were increased in tumor tissues compared with adjacent normal tissues $(\mathrm{P}<0.05$; Fig. 1A-D). Similar results were obtained by western blot analysis $(\mathrm{P}<0.05 ;$ Fig. 1E, F). The tumor stage and grade were classified according to the TNM staging system of the UICC guidelines. The level of EZH2 expression in tumor tissues was significantly increased in the higher TNM stages (T2-T4) compared with the lower stages (Ta-T1; Table I). The EZH2 levels were associated with the tumor histological stage $(\mathrm{P}=0.026)$ and tumor histological grade $(\mathrm{P}<0.001)$. These results indicated that the expression level of EZH2 was closely associated with the progression of bladder cancer.

UNC1999 inhibits the proliferation and migration of the bladder cancer cell lines E-J and 5637. The MTT, apoptosis, wound-healing and cell migration assays were used to investigate the effects of the EZH2 inhibitor UNC1999 on the proliferation and migration of bladder cancer cell lines E-J and 5637 (cells in the control groups were treated with an equal volume of DMSO; Fig. 2). The MTT assay revealed that treatment with UNC1999 inhibited the proliferation of the bladder cancer cell lines in a dose- and time-dependent manner (Fig. 2A and B). UNC1999 exhibited the greatest inhibitory effect at a final concentration of $100 \mu \mathrm{M}$ and an incubation period of 72 and $120 \mathrm{~h}$. The difference between the 72 and $120 \mathrm{~h}$ incubation periods was not statistically significant ( $\mathrm{P}>0.05)$. Therefore, the bladder cancer cell lines were treated with $100 \mu \mathrm{M}$ UNC1999 for $72 \mathrm{~h}$ in subsequent experiments. Apoptosis analysis revealed that UNC1999 induced significant apoptosis in E-J and 5637 cells (Fig. 2C-E).

Cell migration is a central process in the evolution and progression of tumors (23). The wound healing and Transwell migration assays were used to investigate the effects of UNC1999 on the cell migration of bladder cancer cell lines. E-J and 5637 cells treated with UNC1999 exhibited decreased migration compared with the control group which was treated with DMSO $(\mathrm{P}<0.05)$ in the wound healing assay (Fig. 2F and G). Similar results were obtained with the Transwell assay, where a fewer number of cells treated with UNC1999 migrated into the lower chamber compared with control cells $(\mathrm{P}<0.01$; Fig. $2 \mathrm{H}$ and $\mathrm{I})$. The data obtained from the apoptosis and migration assays suggested that UNC1999 reduced the proliferation and migration of the bladder cancer cell lines.

EZH2 inhibition suppresses bladder cancer via blocking of the JAK2/STAT3 signaling pathway. The JAK2/STAT3 signaling pathway controls the invasive and aggressive phenotype of cancer cells (24). The current study investigated the effect of EZH2 on the phosphorylation levels of JAK2 and STAT3, as the phosphorylation is required for the activity of 
A

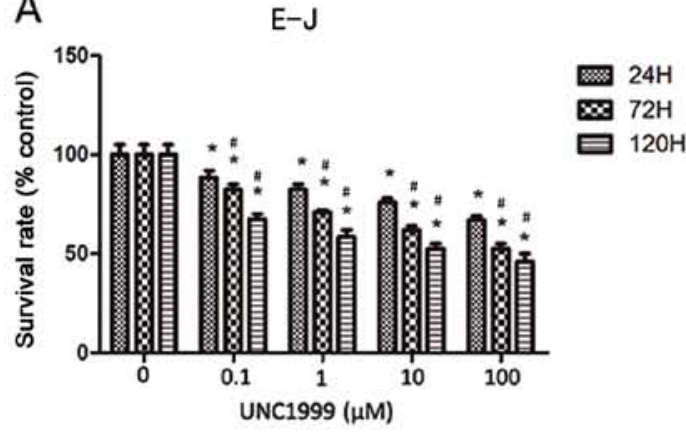

C

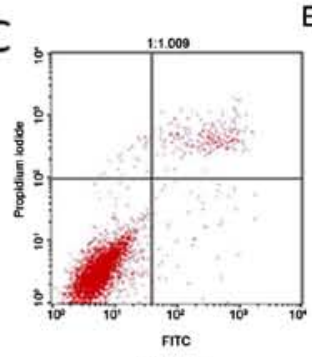

E-J
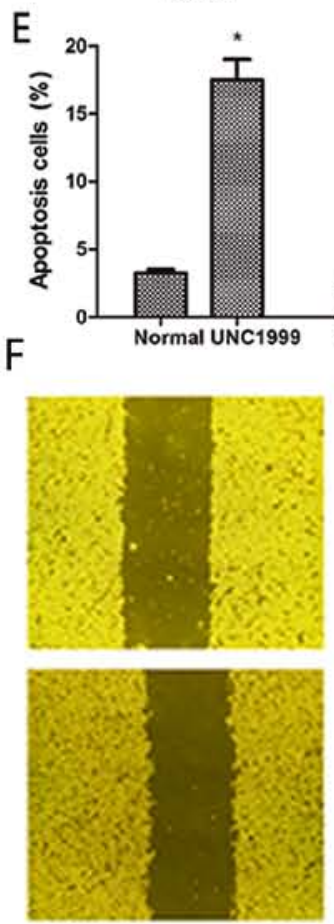

Normal

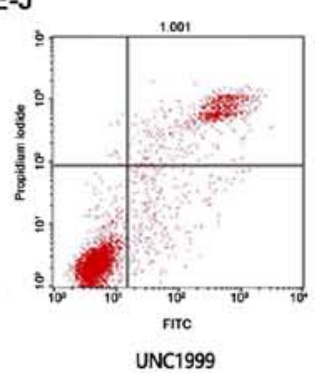

Normal UNC1999

E-J
$\mathrm{Oh}$
B
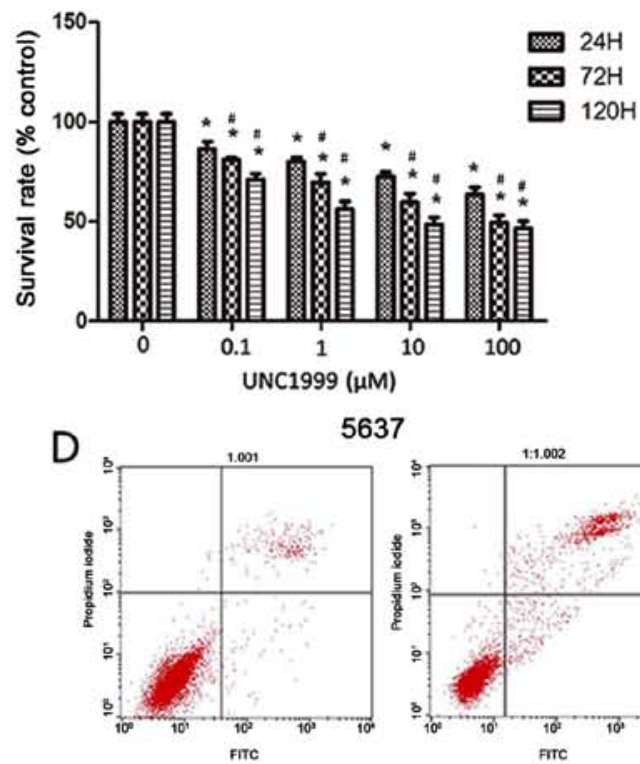

Normal
5637

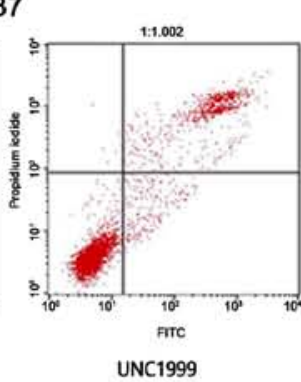

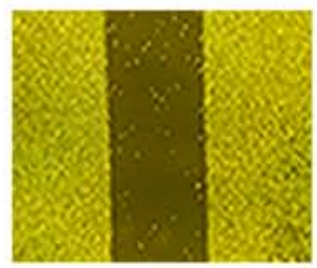

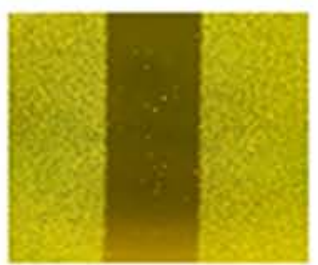

$24 \mathrm{~h}$

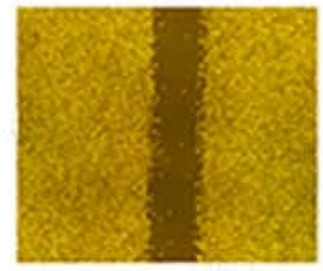

Normal

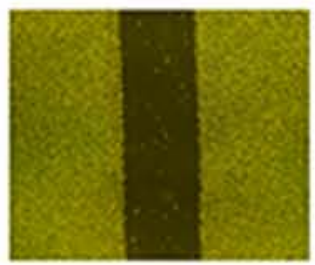

UNC1999
G

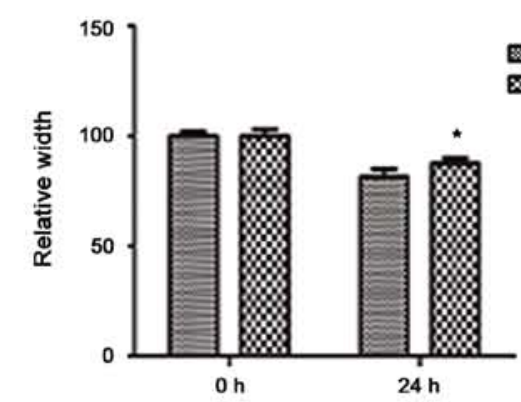

EJ

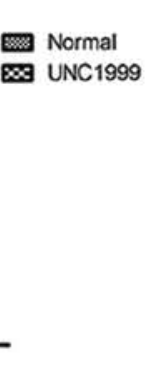

5637

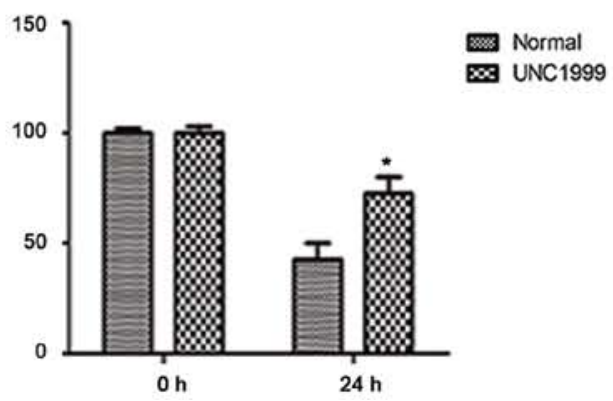

Figure 2. Inhibition of EZH2 suppresses bladder cancer cell migration and viability. The viability of (A) E-J and (B) 5637 cells treated with UNC1999 was measured using the MTT assay. (C) Results of flow cytometry for E-J cells. (D) Results of flow cytometry for 5637 cells. (E) Quantitative analysis of the apoptotic cells. (F) The migration of bladder cancer cells treated with UNC1999. Magnification, x200. (G) The wound healing assay was used to measure the migration ability of bladder cancer cells treated with UNC1999. ${ }^{*} \mathrm{P}<0.01$ vs. normal group (treated with DMSO); ${ }^{\text {\# }}<<0.05$ vs. $24 \mathrm{H}$ group. 
$\mathrm{H}$

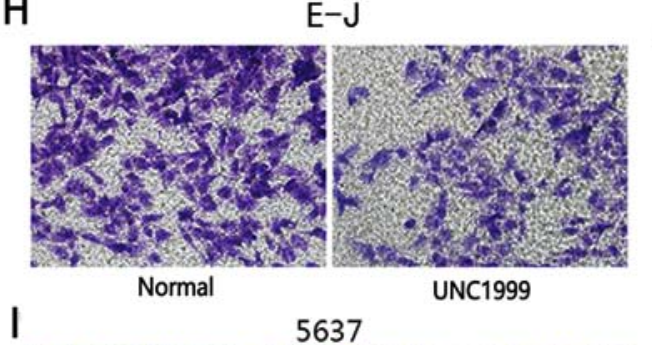

I

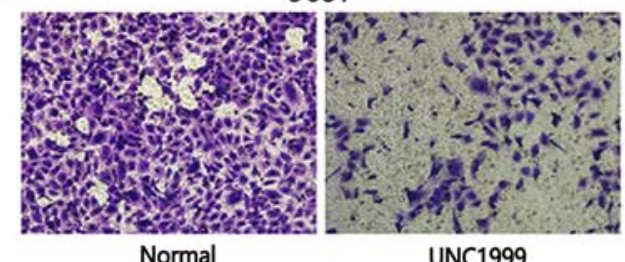

J

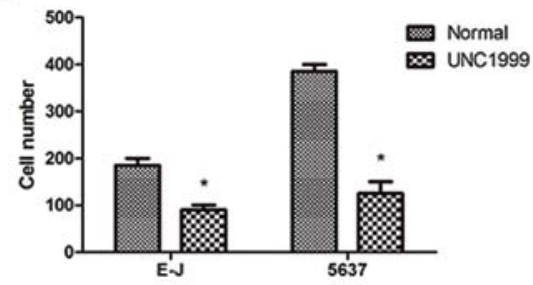

Figure 2. Continued. Inhibition of EZH2 suppresses bladder cancer cell migration and viability. (H) Migration of E-J cells treated with UNC1999 was measured using a Transwell assay. Magnification, x400. (I) Migration of 5637 cells treated with UNC1999 was measured using a Transwell assay. Magnification, x400. (J) Quantitative analysis of the migration of bladder cancer cells. ${ }^{*} \mathrm{P}<0.01$ vs. normal group (treated with DMSO); ${ }^{*} \mathrm{P}<0.05$ vs. $24 \mathrm{H}$ group. EZH2, enhancer of zeste 2 polycomb repressive complex 2 subunit.

A

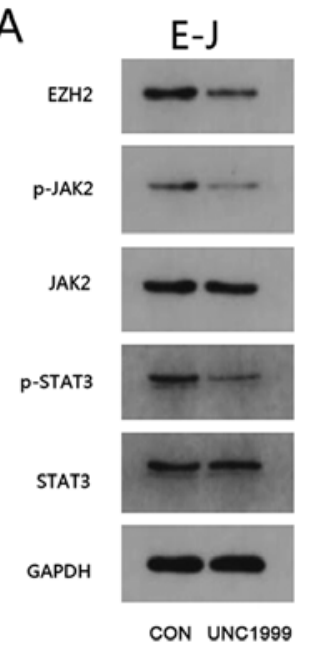

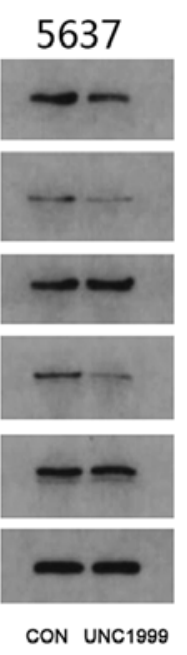

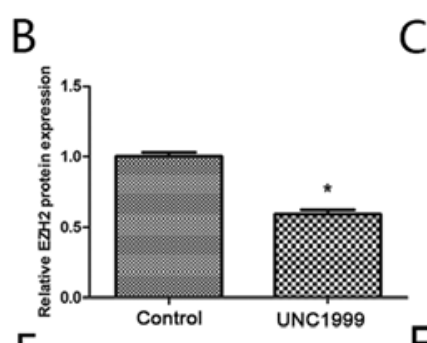

C

E

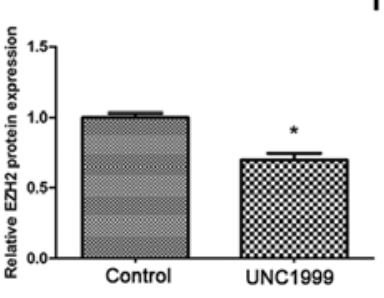

$F$
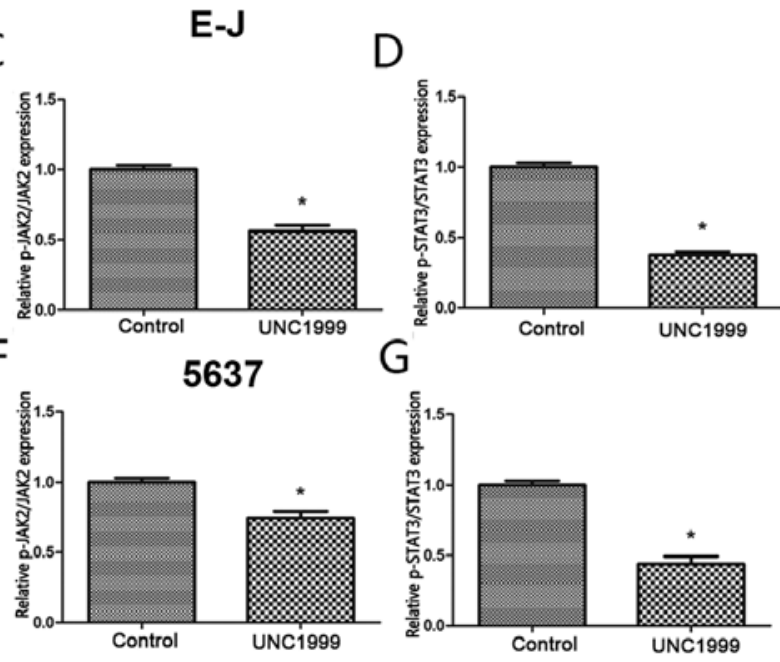

Figure 3. UNC1999 blocked the JAK2/STAT3 signaling pathway in vitro. (A) The levels of EZH2, JAK2, STAT3 and their phosphorylated forms in E-J and 5637 cells were analyzed by western blotting. The quantitative data of (B) EZH2, (C) p-JAK2 and (D) p-STAT3 in E-J cells treated with or without UNC1999. The quantitative data of (E) EZH2, (F) p-JAK2 and (G) p-STAT3 in 5637 cells treated with or without UNC1999. *P<0.01 vs. control group. EZH2, enhancer of zeste 2 polycomb repressive complex 2 subunit; JAK2, janus kinase 2; p-, phosphorylated-; JAK2, janus kinase 2; STAT3, signal transducer and activator of transcription 3.

the JAK2/STAT3 signaling pathway. Constitutive p-JAK2 and p-STAT3 levels were decreased in E-J and 5637 cells treated with UNC1999 compared with controls ( $\mathrm{P}<0.05$; Fig. 3). These data suggested that inhibition of EZH2 may regulate the activation of the JAK2/STAT3 signaling pathway in bladder cancer cell lines.

UNC1999 inhibits bladder cancer growth in vivo. To further explore the antitumor activity of UNC1999 in vivo, E-J tumor xenografts were analyzed. UNC1999 exhibited significant antitumor activity in nude mice bearing E-J tumor xenografts at a dose of $50 \mathrm{mg} / \mathrm{kg}(\mathrm{P}<0.05$; Fig. 4A). In order to examine the mechanism underlying the inhibition of tumor growth by UNC1999 in vivo, the expression levels of JAK2 and STAT3 were measured using western blotting. The expression levels of
JAK2 and STAT3 were significantly decreased in the tumors treated with UNC1999 compared with the control tumors $(\mathrm{P}<0.05$; Fig. 4B-E). These results suggested that UNC1999 inhibited tumor growth in vivo by inhibiting EZH2 and subsequent inhibition of the JAK2/STAT3 signaling pathway.

\section{Discussion}

Bladder cancer is a common cause of cancer-associated mortality worldwide (25). The majority of patients with bladder cancer are diagnosed at an advanced stage of the disease due to a lack of disease-specific makers in the early stages (26). Surgery and chemotherapy are currently the most effective treatments for bladder cancer; however, the prognosis remains poor in the advanced stages and the overall survival rate is 
A

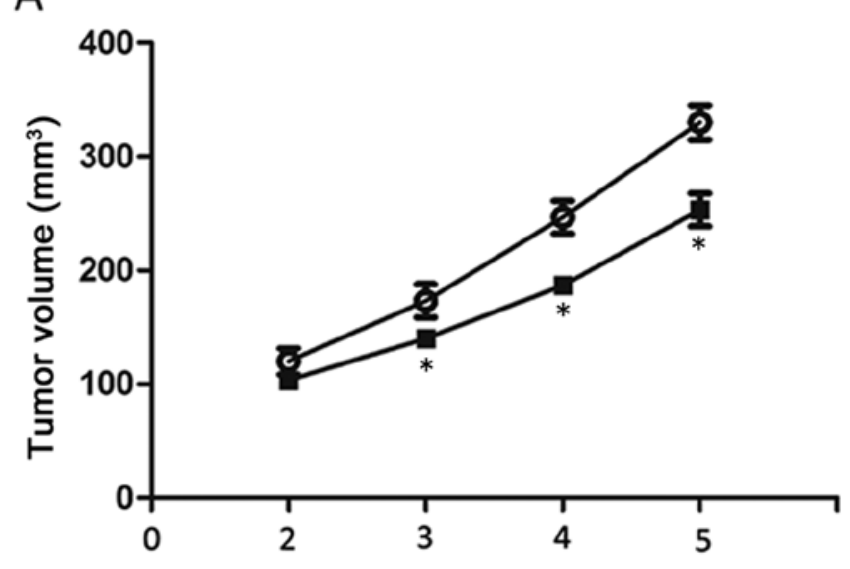

C

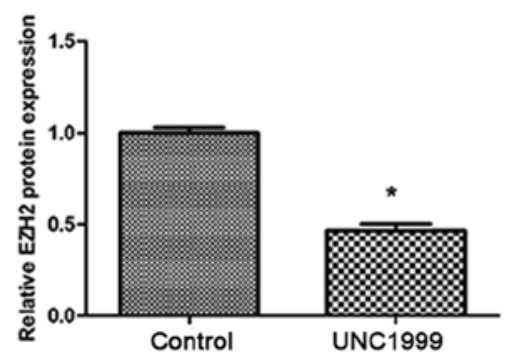

D

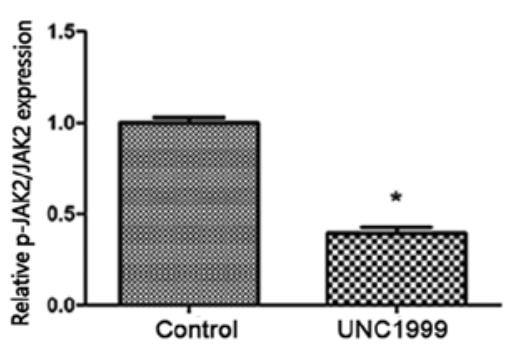

B

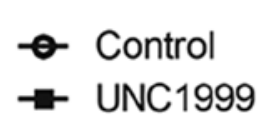

(Weeks)

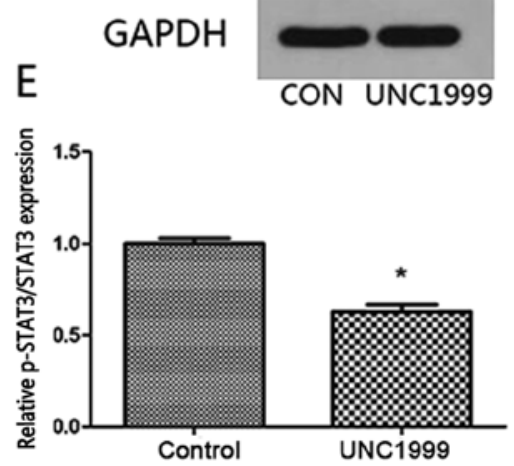

Figure 4. UNC1999 inhibited growth of E-J xenografts and block the JAK2/STAT3 signaling pathway in vivo. (A) Graphs presenting the average tumor volumes of E-J xenografts treated with or without UNC1999. (B) The levels of EZH2, JAK2, STAT3 and their phosphorylated forms were analyzed by western blotting. The quantitative data of (C) EZH2, (D) JAK2 and (E) STAT3 and their phosphorylated forms in E-J xenografts treated with or without UNC1999. ${ }^{*} \mathrm{P}<0.01$ vs. control group. EZH2, enhancer of zeste 2 polycomb repressive complex 2 subunit; JAK2, janus kinase 2; p-, phosphorylated-; JAK2, janus kinase 2; STAT3, signal transducer and activator of transcription 3.

unsatisfactory (27). A number of oncogenes have been identified in recent years, but the molecular mechanisms underlying tumorigenesis of bladder cancer remain unknown (28). Therefore, the identification of novel molecular targets in bladder cancer may improve the diagnosis and prognosis of the disease.

$\mathrm{EZH} 2$ is a member of the polycomb repressive complex 2 . EZHZ, together with embryonic ectoderm development and S UZ12 polycomb repressive complex 2 subunit, catalyze the di- and tri-methylation of histone H3 lysine 27 (H3K27), which is essential for embryonic stem cell pluripotency and self-renewal (29). EZH2 is an adhesion protein expressed in a number of organs and tissues, and it can promote cancer metastasis (30). EZH2 has been reported to be a marker of the aggressive stages of prostate cancer (31). The ectopic overexpression of EZH2 can lead to the transformation of normal prostatic cells and the canceration of breast epithelium (32). EZH2 upregulation is a potential tumor biomarker and contributes to tumor progression; therefore, it is deemed to be an oncogene (33). A previous study suggested that EZH2 inhibition by small interfering RNA may decrease cancer cell proliferation, induce cancer cell apoptosis in vitro and decrease breast xenograft growth in vivo (34). Taken together, EZH2 may be a novel target for drug development for different types of cancer.

UNC1999 is a novel S-adenosyl-1-methionine-competitive EZH2 inhibitor that has demonstrated efficacy in different types of cancer, including leukemia, colon cancer and multiple myeloma (35). The results obtained in the present study indicated that the EZH2 inhibitor UNC1999 may reduce cancer cell proliferation and migration, induce bladder cancer cell apoptosis and contribute to the regression of bladder tumor xenografts in mice. Additionally, the present study revealed that EZH2 may be upregulated in bladder cancer and suggested that pharmacological inhibition of EZH2 may be a novel therapeutic strategy in bladder cancer. The increase of intratumoral EZH2 expression, which is associated with bladder cancer progression and poor prognosis, is indicated as an independent prognostic factor for the overall survival time in clinical patients $(15,36,37)$. Lee et al (36) revealed a predictive value of high expression levels of EZH2 for prognosis in bladder tumors, and that the E2F transcription factor 1-EZH2-SUZ12 polycomb repressive complex 2 subunit-driven transcriptional events may regulate cancer aggressiveness and chemoresistance. This highlights the potential applications for intratumoral EZH2 immunostaining in future clinical prognostic stratification and therapeutic interventions.

JAK2 is a vital hormone signaling and intracellular mediator of cytokines (38). JAK2 can activate a series of downstream signaling pathways, including the STAT cascade $(39,40)$. STAT3, a member of the STAT family, may be activated by phosphorylation at the tyrosine residue 705 to regulate the release of growth factors and cytokines in gastric cancer (41). The activation of the JAK2/STAT3 signaling pathway was associated with the growth, migration and metastasis of lung cancer cells (42). Lei et al (43) revealed that the activation of 
the JAK2/STAT3 signaling pathway may promote the proliferation and migration of cancer cells as well as inhibit apoptosis in renal cell carcinoma. The inhibition of JAK2/STAT3 phosphorylation in pancreatic cancer may reduce the proliferation of pancreatic cancer cells in vivo and in vitro (44). In the current study, the western blotting results revealed that the phosphorylation of the JAK2/STAT3 signaling pathway was associated with the proliferation of bladder cancer cells, and the downregulation of EZH2 expression levels was associated with low expression levels of p-JAK 2 in vivo and in vitro. These results suggested that EZH2 activity may serve an important role in bladder cancer development.

In summary, the present study demonstrated that UNC1999 decreased the proliferation, and migration of bladder cancer cells and increased apoptosis by inhibiting the activity of EZH2. Furthermore, the inhibition of EZH2 activity was associated with inhibition of the JAK2/STAT3 signaling pathway. Thus, EZH2 may be a potential therapeutic target for the treatment of patients with bladder cancer.

The present study had a number of limitations. UNC1999 is the first orally bioavailable inhibitor that has high in vitro potency for wild-type and mutant EZH2 as well as EZH1. EZH1 is a H3K27 methyltransferase that shares $96 \%$ sequence identity with EZH2 in their respective catalytic domains (45). To the best of our knowledge, there are no previously published studies suggesting that EZH1 may be associated with tumor progression. Additionally, the current study did not investigate the mechanism of EZH1 in bladder cancer. Future experiments knocking out or silencing EZH2 may remove the potentially confounding results obtained by inhibiting EZH1. The results of these studies may be compared with the results obtained with or without UNC1999 inhibition. The current study suggested that the JAK2/STAT3 signaling pathway may serve a key role in the in the carcinogenic mechanism of EZH2 in bladder cancer. However, it is likely that several other pathways may be involved in the progression of bladder cancer. Long non-coding RNA, microRNA, circular RNA and exosomes may also be implicated. Future studies investigating other possible mechanisms underlying the progression of bladder cancer are required.

\section{Acknowledgements}

Not applicable.

\section{Funding}

No funding was received.

\section{Availability of data and materials}

The datasets produced during and/or analyzed during the current study are available from the corresponding author on reasonable request.

\section{Authors' contributions}

ZC, JG and LW conceived and designed the experiments. ZC and YD performed the experiments. XL, HC, MW, XW and XDW analyzed the data, contributed to the interpretation of results obtained and wrote the manuscript. JG edited the final draft of the manuscript. All authors read and approved the final manuscript.

\section{Ethics approval and consent to participate}

The present study was approved by The Ethics Committee of Renmin Hospital of Wuhan University (Wuhan, China), and written informed consent was obtained from all participants. The Institutional Animal Care and Use Committee of Wuhan University approved the experimental protocols and supervised the care of animals and experimental procedures.

\section{Patient consent for publication}

Not applicable.

\section{Competing interests}

The authors declare that they have no competing interests.

\section{References}

1. Siegel RL, Miller KD, Fedewa SA, Ahnen DJ, Meester RGS, Barzi A and Jemal A: Colorectal cancer statistics, 2017. CA Cancer J Clin 67: 177-193, 2017.

2. Siegel RL, Miller KD and Jemal A: Cancer statistics, 2015. CA Cancer J Clin 65: 5-29, 2015.

3. Zhang Z, Zhang G and Kong C: Targeted inhibition of polo-like kinase 1 by a novel small-molecule inhibitor induces mitotic catastrophe and apoptosis in human bladder cancer cells. J Cell Mol Med 21: 758-767, 2017.

4. Bray F, Ferlay J, Soerjomataram I, Siegel RL, Torre LA and Jemal A: Global cancer statistics 2018: GLOBOCAN estimates of incidence and mortality worldwide for 36 cancers in 185 countries. CA Cancer J Clin 68: 394-424, 2018.

5. Yan K, Zhang C, Feng J, Hou L, Yan L, Zhou Z, Liu Z, Liu C, Fan Y, Zheng B and Xu Z: Induction of G1 cell cycle arrest and apoptosis by berberine in bladder cancer cells. Eur J Pharmacol 661: 1-7, 2011.

6. Daneshmand S, Patel S, Lotan Y, Pohar K, Trabulsi E, Woods M, Downs T, Huang W, Jones J, O'Donnell M, et al: Efficacy and safety of blue light flexible cystoscopy with hexaminolevulinate in the surveillance of bladder cancer: A phase III, comparative, multicenter study. J Urol 199: 1158-1165, 2018.

7. Sun J, Yu M, Lu Y, Thakur C, Chen B, Qiu P, Zhao H and Chen F: Carcinogenic metalloid arsenic induces expression of mdig oncogene through JNK and STAT3 activation. Cancer Lett 346: 257-263, 2014.

8. Bromberg JF, Wrzeszczynska MH, Devgan G, Zhao Y, Pestell RG, Albanese C and Darnell JE Jr: Stat 3 as an oncogene. Cell 98: 295-303, 1999.

9. Devarajan E and Huang S: STAT3 as a central regulator of tumor metastases. Curr Mol Med 9: 626-633, 2009.

10. Wagner KU and Schmidt JW: The two faces of Janus kinases and their respective STATs in mammary gland development and cancer. J Carcinog 10: 32, 2011.

11. Gu W, Zhang E, Song L, Tu L, Wang Z, Tian F, Aikenmu K, Chu $G$ and Zhao J: Long noncoding RNA HOXD-AS1 aggravates osteosarcoma carcinogenesis through epigenetically inhibiting p57 via EZH2. Biomed Pharmacother 106: 890-895, 2018.

12. Pasini D, Bracken AP, Hansen JB, Capillo M and Helin K: The polycomb group protein Suz12 is required for embryonic stem cell differentiation. Mol Cell Biol 27: 3769-3779, 2007.

13. Chamberlain SJ, Yee D and Magnuson T: Polycomb repressive complex 2 is dispensable for maintenance of embryonic stem cell pluripotency. Stem Cells 26: 1496-1505, 2008.

14. Labbe DP, Sweeney CJ, Brown M, Galbo P, Rosario S, Wadosky KM, Ku SY, Sjöström M, Alshalalfa M, Erho N, et al: TOP $2 \mathrm{~A}$ and $\mathrm{EZH} 2$ provide early detection of an aggressive prostate cancer subgroup. Clin Cancer Res 23: 7072-7083, 2017. 
15. Liu D, Li Y, Luo G, Xiao X, Tao D, Wu X, Wang M, Huang C, Wang L, Zeng F and Jiang G: LncRNA SPRY4-IT1 sponges miR-101-3p to promote proliferation and metastasis of bladder cancer cells through up-regulating EZH2. Cancer Lett 388: 281-291, 2017.

16. Wang Y, Chen Y, Geng H, Qi C, Liu Y and Yue D: Overexpression of $\mathrm{YB} 1$ and $\mathrm{EZH} 2$ are associated with cancer metastasis and poor prognosis in renal cell carcinomas. Tumour Biol 36: 7159-7166, 2015.

17. Reijm EA, Timmermans AM, Look MP, Meijer-van GM, Stobbe CK, van Deurzen CH, Martens JW, Sleijfer S, Foekens JA, Berns PM and Jansen MP: High protein expression of EZH2 is related to unfavorable outcome to tamoxifen in metastatic breast cancer. Ann Oncol 25: 2185-2190, 2014.

18. Shen H, Morrison CD, Zhang J, Underwood W III, Yang N, Frangou C, Eng K, Head K, Bollag RJ, Kavuri SK, et al: 6p22.3 amplification as a biomarker and potential therapeutic target of advanced stage bladder cancer. Oncotarget 4: 2124-2134, 2013.

19. Li Z, Wang Y, Qiu J, Li Q, Yuan C, Zhang W, Wang D, Ye J, Jiang H, Yang $\mathrm{J}$ and Cheng $\mathrm{J}$ : The polycomb group protein EZH2 is a novel therapeutic target in tongue cancer. Oncotarget 4: 2532-2549, 2013.

20. Magers MJ, Lopez-Beltran A, Montironi R, Williamson SR, Kaimakliotis HZ and Cheng L: Staging of bladder cancer. Histopathology 74: 112-134, 2019.

21. Livak KJ and Schmittgen TD: Analysis of relative gene expression data using real-time quantitative PCR and the 2(-Delta Delta C(T)) method. Methods 25: 402-408, 2008

22. Moon JH, Hong SW, Kim JE, Shin JS, Kim JS, Jung SA, Ha SH, Lee S, Kim J, Lee DH, et al: Targeting $\beta$-catenin overcomes MEK inhibition resistance in colon cancer with KRAS and PIK3CA mutations. Br J Cancer: 4 April, 2019.

23. Chen J, Weihs D and Vermolen FJ: A model for cell migration in non-isotropic fibrin networks with an application to pancreatic tumor islets. Biomech Model Mechanobiol 17: 367-386, 2018

24. Zhao JM, Cheng W, He XG, Liu YL, Wang FF and Gao YF: Long non-coding RNA PICART1 suppresses proliferation and promotes apoptosis in lung cancer cells by inhibiting JAK2/STAT3 signaling. Neoplasma 65: 779-789, 2018.

25. Torre LA, Bray F, Siegel RL, Ferlay J, Lortet-Tieulent J and Jemal A: Global cancer statistics, 2012. CA Cancer J Clin 65: 87-108, 2015.

26. Dehayni Y, Tetou M, Khdach Y, Janane A, Alami M and Ameur A: Prognostic of older age for patients with invasive-muscle-bladder cancer and treated by radical cystectomy. Prog Urol 28: 166-172, 2018 (In French).

27. Racioppi M, D'Agostino D, Totaro A, Pinto F, Sacco E, D'Addessi A, Marangi F, Palermo G and Bassi PF: Value of current chemotherapy and surgery in advanced and metastatic bladder cancer. Urol Int 88: 249-258, 2012.

28. Hammerle M, Gutschner T, Uckelmann H, Ozgur S, Fiskin E, Gross M, Skawran B, Geffers R, Longerich T, Breuhahn K, et al: Posttranscriptional destabilization of the liver-specific long noncoding RNA HULC by the IGF2 mRNA-binding protein 1 (IGF2BP1). Hepatology 58: 1703-1712, 2013.

29. Muller J, Hart CM, Francis NJ, Vargas ML, Sengupta A, Wild B, Miller EL, O'Connor MB, Kingston RE and Simon JA: Histone methyltransferase activity of a drosophila polycomb group repressor complex. Cell 111: 197-208, 2002.

30. Shahabipour F, Caraglia M, Majeed M, Derosa G, Maffioli P and Sahebkar A: Naturally occurring anti-cancer agents targeting EZH2. Cancer Lett 400: 325-335, 2017.

31. Varambally S, Dhanasekaran SM, Zhou M, Barrette TR, Kumar-Sinha C, Sanda MG, Ghosh D, Pienta KJ, Sewalt RG, Otte AP, et al: The polycomb group protein EZH2 is involved in progression of prostate cancer. Nature 419: 624-629, 2002.

32. Karanikolas BD, Figueiredo ML and Wu L: Polycomb group protein enhancer of zeste 2 is an oncogene that promotes the neoplastic transformation of a benign prostatic epithelial cell line. Mol Cancer Res 7: 1456-1465, 2009.
33. Yamamoto I, Nosho K, Kanno S, Igarashi H, Kurihara H, Ishigami K, Ishiguro K, Mitsuhashi K, Maruyama R, Koide $\mathrm{H}$, et al: $\mathrm{EZH} 2$ expression is a prognostic biomarker in patients with colorectal cancer treated with anti-EGFR therapeutics. Oncotarget 8: 17810-17818, 2017.

34. Gonzalez ME, Li X, Toy K, DuPrie M, Ventura AC, Banerjee M, Ljungman M, Merajver SD and Kleer CG: Downregulation of EZH2 decreases growth of estrogen receptor-negative invasive breast carcinoma and requires BRCA1. Oncogene 28: 843-853, 2009.

35. Xu B, On DM, Ma A, Parton T, Konze KD, Pattenden SG, Allison DF, Cai L, Rockowitz S, Liu S, et al: Selective inhibition of EZH2 and EZH1 enzymatic activity by a small molecule suppresses MLL-rearranged leukemia. Blood 125: 346-357, 2015.

36. Lee SR, Roh YG, Kim SK, Lee JS, Seol SY, Lee HH, Kim WT, Kim WJ, Heo J, Cha HJ, et al: Activation of EZH2 and SUZ12 regulated by $\mathrm{E} 2 \mathrm{~F} 1$ predicts the disease progression and aggressive characteristics of bladder cancer. Clin Cancer Res 21: 5391-5403, 2015

37. Zhang S, Zhong G, He W, Yu H, Huang J and Lin T: lncRNA Up-regulated in nonmuscle invasive bladder cancer facilitates tumor growth and acts as a negative prognostic factor of recurrence. J Urol 196: 1270-1278, 2016.

38. Xu B, Chen X, Tan J and Xu X: Effect of AG490 on JAK2/STAT3 signaling pathway in human retinoblastoma HXO-RB44 cell lines. Zhong Nan Da Xue Xue Bao Yi Xue Ban 43: 1061-1067, 2018 (In Chinese).

39. Heinrich PC, Behrmann I, Haan S, Hermanns HM, Muller-Newen $G$ and Schaper F: Principles of interleukin (IL)-6-type cytokine signalling and its regulation. Biochem J 374: $1-20,2003$

40. Zheng L, Chen J, Zhou Z and He Z: Knockdown of long non-coding RNA HOXD-AS1 inhibits gastric cancer cell growth via inactivating the JAK2/STAT3 pathway. Tumour Biol 39: 1010428317705335,2017

41. Kim MJ, Nam HJ, Kim HP, Han SW, Im SA, Kim TY, Oh DY and Bang YJ: OPB-31121, a novel small molecular inhibitor, disrupts the JAK2/STAT3 pathway and exhibits an antitumor activity in gastric cancer cells. Cancer Lett 335: 145-152, 2013.

42. Song Y, Kong L, Sun B, Gao L, Chu P, Ahsan A, Qaed E, Lin Y, Peng J, Ma X, et al: Induction of autophagy by an oleanolic acid derivative, SZC017, promotes ROS-dependent apoptosis through Akt and JAK2/STAT3 signaling pathway in human lung cancer cells. Cell Biol Int 41: 1367-1378, 2017.

43. Lei J, Xiao JH, Zhang SH, Liu ZQ, Huang K, Luo ZP, Xiao XL and Hong ZD: Non-coding RNA 886 promotes renal cell carcinoma growth and metastasis through the Janus kinase 2/signal transducer and activator of transcription 3 signaling pathway. Mol Med Rep 16: 4273-4278, 2017.

44. Zhang Z, Wang F, Du C, Guo H, Ma L, Liu X, Kornmann M, Tian X and Yang Y: BRM/SMARCA2 promotes the proliferation and chemoresistance of pancreatic cancer cells by targeting JAK2/STAT3 signaling. Cancer Lett 402: 213-224, 2017.

45. Yang X, Li F, Konze KD, Meslamani J, Ma A, Brown PJ, Zhou MM, Arrowsmith CH, Kaniskan HÜ, Vedadi M and Jin J: Structure-activity relationship studies for enhancer of zeste homologue 2 (EZH2) and enhancer of zeste homologue 1 (EZH1) inhibitors. J Med Chem 59: 7617-7633, 2016.

This work is licensed under a Creative Commons Attribution-NonCommercial-NoDerivatives 4.0 International (CC BY-NC-ND 4.0) License. 